\title{
Anisotropic Left-Handed and $\mu$-Negative Slab Waveguides: Physics and Device Applications
}

\author{
Hamidreza Salehi, Sujeet K. Chaudhuri, and Raafat R. Mansour \\ Department of Electrical and Computer Engineering, University of Waterloo, Waterloo, ON, Canada N2L $3 G 5$
}

Received 25 April 2007; Revised 27 June 2007; Accepted 1 October 2007

Recommended by Stefan A. Maier

We study the properties of various anisotropic left-handed slab waveguides. The analysis is extended to anisotropic $\mu$-negative slab waveguides. The possible existence of the plasmon modes in various anisotropic slab waveguide configurations is discussed. An FDTD program is developed to investigate the potential device applications of these anisotropic structures. A new signal detector and a two-channel harmonic separator multiplexer are designed employing the $\mu$-negative slab waveguide.

Copyright ( $) 2007$ Hamidreza Salehi et al. This is an open access article distributed under the Creative Commons Attribution License, which permits unrestricted use, distribution, and reproduction in any medium, provided the original work is properly cited.

\section{INTRODUCTION}

The concept of a material with simultaneously negative permittivity and permeability, first studied by Veselago in 1968 [1], has attracted physics and engineering communities in recent years. These structures, which exhibit extraordinary properties not generally found in nature, are referred to as negative index materials (NIMs) or left-handed materials (LHMs).

Different research groups have tried to artificially realize these structures, employing subwavelength periodic structures [2-6]. With the possibility of physically realizing negative index materials, it is important to investigate the unusual properties of these composite structures and their possible device applications in the microwave and optical frequency ranges. The unusual properties of left-handed slab waveguides (LHSWs), where a left-handed layer is sandwiched between positive index layers, have been studied in the literature [7-9].

The existence of plasmon modes in LHSWs can be utilized in various device applications, provided these structures can be realized. The LHSW structure requires negative permeability in both normal and transverse directions, and negative primitivity in the transverse direction. From a realization point of view, it is practically easier to realize an LHM, that only requires negative permeability in one direction. This is due to polarization dependency of split-ring resonator structures, which are widely used to realize left-handed composites.
In this paper, we study the guided mode properties of the anisotropic LHSWs, with the aim of finding a structure configuration that supports the plasmon modes. Potential device applications of anisotropic LHSWs, that employ field localization of the plasmon modes, are explored.

To study various device applications of LHSWs, a finite-difference time-domain (FDTD) program is developed to numerically study different devices that incorporate anisotropic slab waveguides. Berenger's perfectly matched layer (BPML) is chosen to truncate the FDTD grid [10]. The Drude dispersion formulation is used to model the permittivity and permeability of the left-handed media. Different proposed device applications of LHSW such as a new signal detector and a two-channel harmonic separator multiplexer are examined by using the developed FDTD code.

\section{ANISOTROPIC LEFT-HANDED SLAB WAVEGUIDES}

The analysis of the left-handed slab waveguides (LHSWs) in [7-9] indicated that the structure can support guided modes with imaginary transverse wavenumbers, referred to as plasmon modes. The energy of these plasmon modes is concentrated at the interfaces of the left-handed core and the dielectric cladding. This characteristic of the plasmon modes suggests that an LHSW can have potential applications in signal detection devices. In an isotropic LHSW, the left-handed layer exhibits negative permeability in both $\hat{x}$ and $\hat{z}$ directions, that is, $\mu_{x x}<0$ and $\mu_{z z}<0$. In practice, it is relatively difficult to realize a left-handed composite that exhibits 


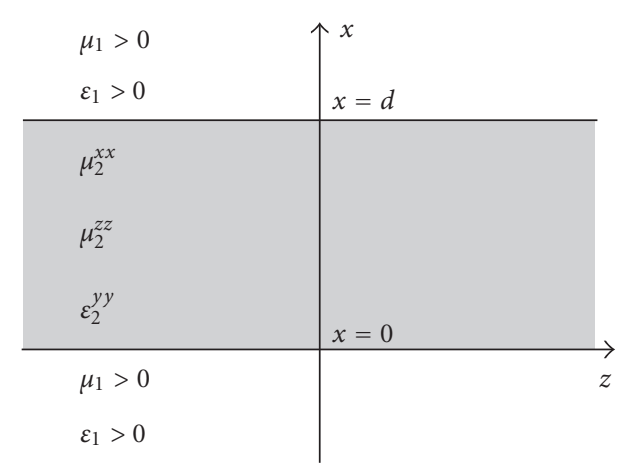

FIGURE 1: Anisotropic $\mu$-negative slab waveguide (SW) of thickness $d$ sandwiched between two dielectric layers.

negative permeability in two directions using the existing structures, specially at optical frequencies [11-17].

In this section, to comply with the practical limitations of realizing an LHSW, the possibility of realizing an anisotropic $\mu$-negative slab waveguide that can support guided modes with imaginary transverse wavenumber is investigated. The anisotropic $\mu$-negative slab waveguide (SW) is considerably simpler to fabricate since the composite requires to exhibit negative permeability only in one direction. The schematic of an anisotropic $\mu$-negative slab waveguide is shown in Figure 1. For the TE polarized waves, with the electric field in the $\hat{y}$ direction and the magnetic fields in the $\hat{x}$ and $\hat{z}$ directions, Maxwell equations are given by

$$
\begin{gathered}
\frac{\partial H_{x}}{\partial z}-\frac{\partial H_{z}}{\partial x}=j \omega \epsilon^{y y} E_{y}, \\
\frac{\partial E_{y}}{\partial z}=j \omega \mu^{x x} H_{x}, \\
\frac{\partial E_{y}}{\partial x}=-j \omega \mu^{z z} H_{z} .
\end{gathered}
$$

The wave equation in the cladding layer that surrounds the left-handed core remains unchanged because the cladding still consists of isotropic dielectric material. By combining (1), the wave equation in the anisotropic $\mu$-negative layer of the slab waveguide is

$$
\frac{\partial^{2} E_{y}(x)}{\partial x^{2}}+\left(\omega^{2} \mu_{2}^{z z} \epsilon_{2}^{y y}-\frac{\mu_{2}^{z z}}{\mu_{2}^{x x}} \beta^{2}\right) E_{y}(x)=0,
$$

where $\beta$ is the propagation constant in the $z$ direction. For the anisotropic LHSW to support the plasmon modes, the derivative of the electric field with respect to $x, \partial E_{y}(x) / \partial x$, should be discontinuous at $x=0$ and $x=d$. The boundary conditions at $x=0$ and $x=d$ require that $H_{z}(x=$ $\left.0^{-}\right)=H_{z}\left(x=0^{+}\right)$, and $H_{z}\left(x=d^{-}\right)=H_{z}\left(x=d^{+}\right)$, where $H_{z}=\left(j / \omega \mu_{2}^{z z}\right)\left(\partial E_{y}(x) / \partial x\right)$. The discontinuity of $\partial E_{y}(x) / \partial x$ requirement along with the continuity of $H_{z}$ at the interfaces indicates that for a left-handed slab waveguide to support plasmon modes, $\mu_{2}^{z z}$ needs to be negative. The wave equation of an LHSW with $\mu_{2}^{z z}<0, \mu_{2}^{x x}>0$, and $\epsilon_{2}^{y y}<0$ is

$$
\frac{\partial^{2} E_{y}(x)}{\partial x^{2}}+p^{2} E_{y}(x)=0
$$

where $p^{2}=\omega^{2} \mu_{2}^{z z} \epsilon_{2}^{y y}-\left(\mu_{2}^{z z} / \mu_{2}^{x x}\right) \beta^{2}>0$. The solutions of this differential equation are in the form of $A \exp ( \pm j p x)$, which indicates that such a structure cannot support guided modes with imaginary wavenumbers; that is, (3) does not have any solutions in the form of $A \exp ( \pm h x)$. To overcome this problem, the electric permittivity of the left-handed layer, $\epsilon_{2}^{y y}$, is chosen to be positive. This transforms the structure into a $\mu$-negative structure, which will be studied in the following section.

\section{ANISOTROPIC $\mu$-NEGATIVE SLAB WAVEGUIDE}

The wave equation of the anisotropic $\mu$-negative medium with $\mu_{2}^{z z}<0, \mu_{2}^{x x}>0$, and $\epsilon_{2}^{y y}>0$ can have plasmon-type solutions if $\left(\mu_{2}^{z z} / \mu_{2}^{x x}\right)^{2} \beta^{2}<\omega^{2} \mu_{2}^{z z} \epsilon_{2}^{y y}$. The solutions of the wave equation

$$
\frac{\partial^{2} E_{y}(x)}{\partial x^{2}}-h^{2} E_{y}(x)=0,
$$

where $h^{2}=\omega^{2}\left|\mu_{2}^{z z} \epsilon_{2}^{y y}\right|-(\xi \beta)^{2}>0$ and $\xi^{2}=-\mu_{2}^{z z} / \mu_{2}^{x x}$, are in the form of $E_{y}(0<x<d)=A \exp ( \pm h x)$, revealing that this structure can support plasmon modes. Solving the isotropic wave equation in the cladding and satisfying the electric and magnetic boundary conditions at $x=0$ and $x=d$ show that the propagation constant of the even plasmon mode, $\mathrm{TE}_{\text {even }}^{\text {Plasmon, }}$, is found from the intersection of

$$
\begin{gathered}
v=-\left(\frac{\mu_{1}}{\mu_{2}^{z z}}\right) u \tanh \left(\frac{u}{2}\right), \\
\xi^{2} v^{2}+u^{2}=4 \pi^{2}\left(\frac{d}{\lambda}\right)^{2}\left(\left|\mu_{2}^{z z} \epsilon_{2}^{y y}\right|-\mu_{1} \epsilon_{1} \xi^{2}\right),
\end{gathered}
$$

where $u=h d, v=q d, h=\sqrt{\omega^{2}\left|\mu_{2}^{z z} \epsilon_{2}^{y y}\right|-\xi^{2} \beta^{2}}$, and $q=$ $\sqrt{\beta^{2}-\omega^{2} \mu_{1} \epsilon_{1}}$. The propagation constant of the odd plasmon mode, $\mathrm{TE}_{\text {odd }}^{\text {Plasmon }}$, is similarly found from the intersection of

$$
\begin{gathered}
v=-\left(\frac{\mu_{1}}{\mu_{2}^{z z}}\right) u \operatorname{coth}\left(\frac{u}{2}\right), \\
\xi^{2} v^{2}+u^{2}=4 \pi^{2}\left(\frac{d}{\lambda}\right)^{2}\left(\left|\mu_{2}^{z z} \epsilon_{2}^{y y}\right|-\mu_{1} \epsilon_{1} \xi^{2}\right) .
\end{gathered}
$$

The graphical solution of the plasmon modes, depicted in Figure 2, indicates that the anisotropic $\mu$-negative SW always supports the even plasmon mode while the odd mode is excited if the operating frequency is above a certain cutoff frequency. As the operating frequency increases, the field concentration of plasmon modes at the interfaces of the lefthanded and dielectric materials, the core-cladding interface, increases and the propagation constants of even and odd modes converge towards an identical value. The electric field distribution of both even and odd plasmon modes of the anisotropic $\mu$-negative SW with $\mu_{2}^{z z}<0, \mu_{2}^{x x}>0$, and $\epsilon_{2}^{y y}>0$, is depicted in Figures 3 and 4 , respectively. With $\mu_{2}^{z z}=-1$, $\mu_{2}^{x x}=1$, and $\epsilon_{2}^{y y}=2.25$, the SW also supports guided modes with real transverse wavenumbers similar to a conventional slab waveguide. The propagation constants of these guided 


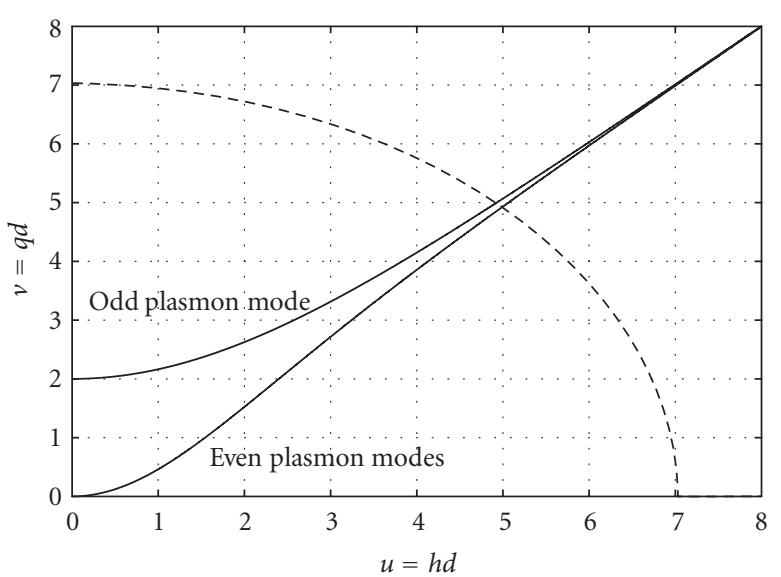

FIgURE 2: Mode diagram of the anisotropic $\mu$-negative slab waveguide (LHSW) with $\mu_{2}^{z z}=-1, \mu_{2}^{x x}=1$, and $\epsilon_{2}^{y y}=2.25(h=$ $\sqrt{\omega^{2}\left|\mu_{2}^{z z} \epsilon_{2}^{y y}\right|-\xi^{2} \beta^{2}}$, and $\left.q=\sqrt{\beta^{2}-\omega^{2} \mu_{1} \epsilon_{1}}\right)$.

modes have $\left(\mu_{2}^{z z} / \mu_{2}^{x x}\right)^{2} \beta^{2}>\omega^{2} \mu_{2}^{z z} \epsilon_{2}^{y y}$, resulting in the following wave equation in the anisotropic $\mu$-negative layer:

$$
\frac{\partial^{2} E_{y}(x)}{\partial x^{2}}+p^{2} E_{y}(x)=0
$$

where $p^{2}=(\xi \beta)^{2}-\omega^{2}\left|\mu_{2}^{z z} \epsilon_{2}^{y y}\right|>0$ and $\xi^{2}=-\left(\mu_{2}^{z z} / \mu_{2}^{x x}\right)$. The solutions of (7) are in the form of $E_{y}(0<x<d)=$ $A \exp ( \pm j p x)$, indicating that the structure also supports guided modes with real transverse wavenumbers. Similarly, solving the isotropic wave equation in the cladding and satisfying the electric and magnetic boundary conditions at $x=0$ and $x=d$ show that the propagation constants of the odd guided modes with real transverse wavenumbers, $\mathrm{TE}_{2 n+1}$, are found from the intersections of

$$
\begin{gathered}
v=\left(\frac{\mu_{1}}{\mu_{2}^{z z}}\right) u \tan \left(\frac{u}{2}\right), \\
u^{2}-\xi^{2} v^{2}=4 \pi^{2}\left(\frac{d}{\lambda}\right)^{2}\left(\mu_{1} \epsilon_{1} \xi^{2}-\left|\mu_{2}^{z z} \epsilon_{2}^{y y}\right|\right),
\end{gathered}
$$

where $u=p d$ and $v=q d$. The propagation constants of the even guided modes, $\mathrm{TE}_{2 n}$, are similarly found from the intersections of

$$
\begin{gathered}
v=-\left(\frac{\mu_{1}}{\mu_{2}^{z z}}\right) u \cot \left(\frac{u}{2}\right), \\
u^{2}-\xi^{2} v^{2}=4 \pi^{2}\left(\frac{d}{\lambda}\right)^{2}\left(\mu_{1} \epsilon_{1} \xi^{2}-\left|\mu_{2}^{z z} \epsilon_{2}^{y y}\right|\right) .
\end{gathered}
$$

The graphical solutions for modes with real transverse number, depicted in Figure 5, indicate that the anisotropic $\mu$ negative SW supports infinite number of discrete guided modes with real transverse wavenumbers at any operating frequencies. The excitation of infinite guided modes with real transverse wavenumbers can be troublesome in the design of any potential device applications that employ the field confinement property of the plasmon modes.

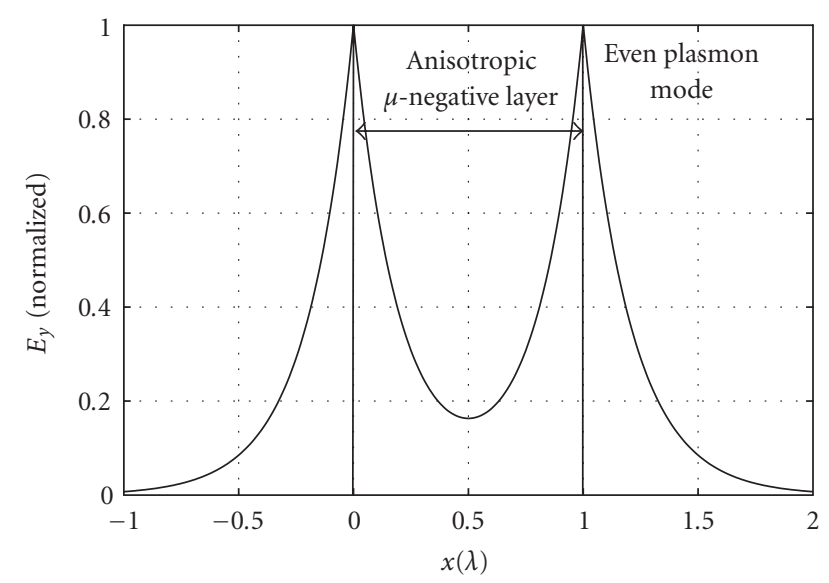

Figure 3: Normalized electric field distribution of the even plasmon modes of the anisotropic $\mu$-negative slab waveguide with $\mu_{2}^{z z}=-1$, $\mu_{2}^{x x}=1$, and $\epsilon_{2}^{y y}=2.25$.

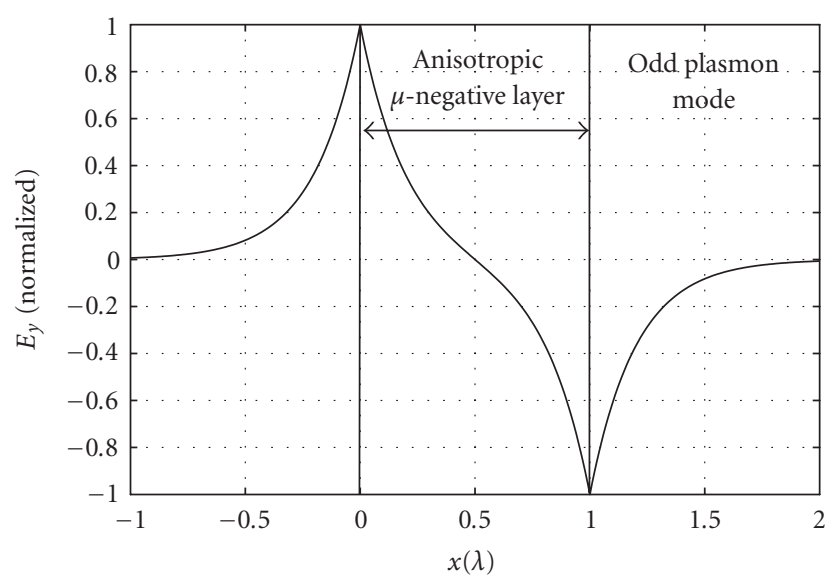

FIGURE 4: Normalized electric field distribution of the odd plasmon modes of the anisotropic $\mu$-negative slab waveguide with $\mu_{2}^{z z}=-1$, $\mu_{2}^{x x}=1$, and $\epsilon_{2}^{y y}=2.25$.

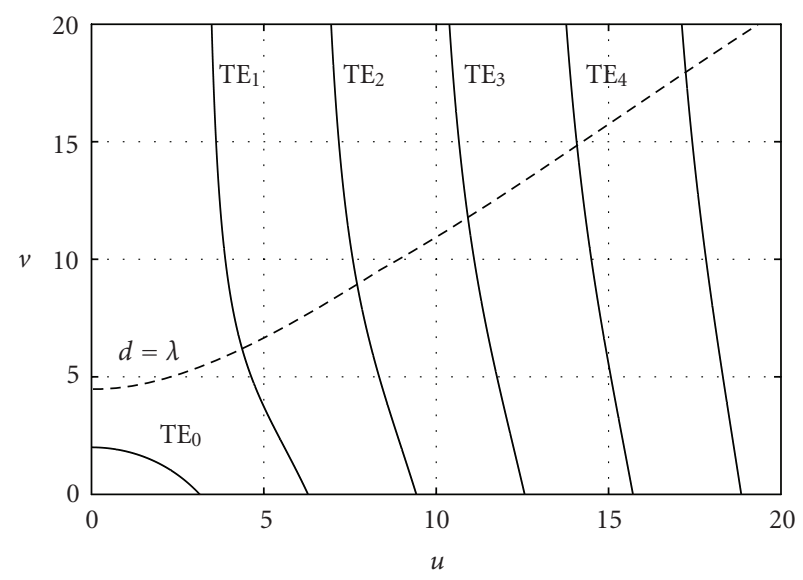

FIGURE 5: Graphical solution for modes with real transverse wavenumbers, $v=q d, u=p d, p=\sqrt{(\xi \beta)^{2}-\omega^{2}\left|\mu_{2}^{z z} \epsilon_{2}^{y y}\right|}$, and $q=\sqrt{\beta^{2}-\omega^{2} \mu_{1} \epsilon_{1}}$. 


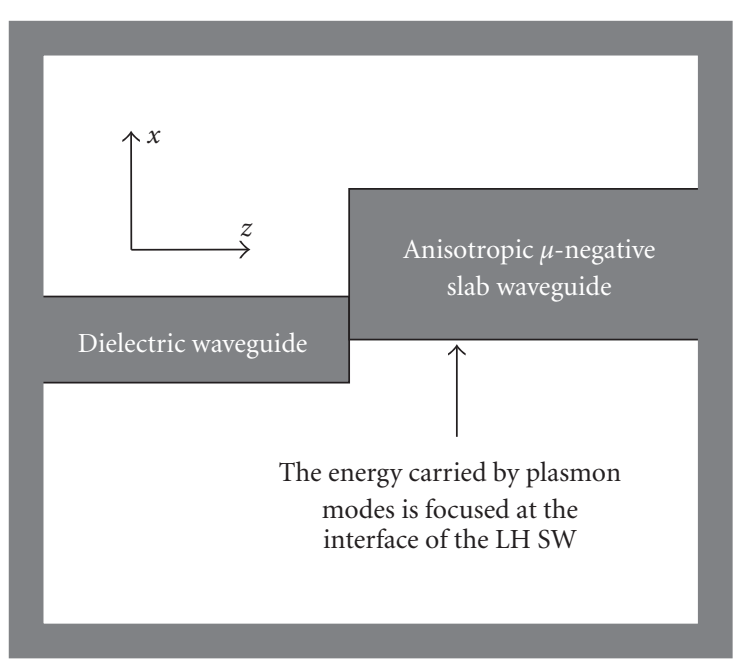

Figure 6: Structure of the proposed signal detector with dielectric waveguide thickness of $0.5 \lambda_{0}$ and anisotropic $\mu$-negative SW of thickness $\lambda_{0}$.

\section{DEVICE APPLICATIONS}

One of the potential device applications of the anisotropic $\mu$-negative SW is in the design of a new signal detector structure. To explain the concept of the proposed signal detector, assume a dielectric slab waveguide that is connected to an anisotropic $\mu$-negative SW as shown in Figure 6. The dielectric slab waveguide is $0.5 \lambda_{0}$ wide and has a dielectric constant of 2.25. The anisotropic $\mu$-negative SW with width of $\lambda_{0}, \mu^{z z}=-1, \mu^{y y}=-1$, and $\epsilon^{y y}=2.25$ is placed in the middle of the dielectric slab waveguide, where the power density of the $\mathrm{TE}_{0}$ mode is maximum. The structure is designed to capture the incoming energy of the $\mathrm{TE}_{0}$ mode and confine it at the interface of the anisotropic $\mu$-negative SW. The plasmon modes of the anisotropic $\mu$-negative SW are then excited and travel along the structure. In a conventional signal detector, the signal detection is performed in the center of the dielectric slab waveguide, where the power density of the $\mathrm{TE}_{0}$ mode is maximum, whereas in the proposed structure, the signal detection is performed at the interface of the anisotropic $\mu$-negative SW, where the power density of the plasmon modes is maximum. The proposed structure localizes the incoming signal at the detector, which in turn increases the sensitivity of the detector.

The proposed signal detector structure, shown in Figure 6, is simulated using the FDTD program developed at the University of Waterloo to simulate various left-handed and anisotropic media. The Drude dispersion formulation is chosen to model the LHM. The permittivity and permeability of the negative medium are modeled by

$$
\begin{aligned}
& \epsilon=\epsilon_{r} \epsilon_{0}\left(1-\frac{\omega_{p}^{2}}{\omega^{2}}\right), \\
& \mu=\mu_{r} \mu_{0}\left(1-\frac{\omega_{p}^{2}}{\omega^{2}}\right) .
\end{aligned}
$$

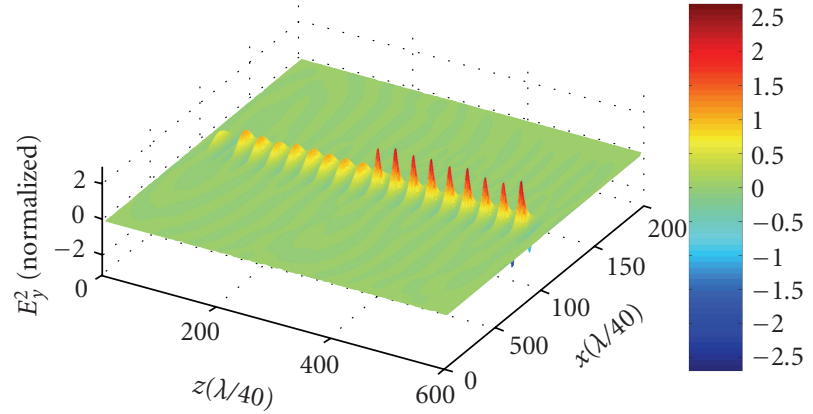

Figure 7: FDTD simulations of the structure in Figure 6, showing the power density distribution of the propagating guided waves in the dielectric slab waveguide and the anisotropic $\mu$-negative slab waveguide (SW) of thickness $\lambda$.

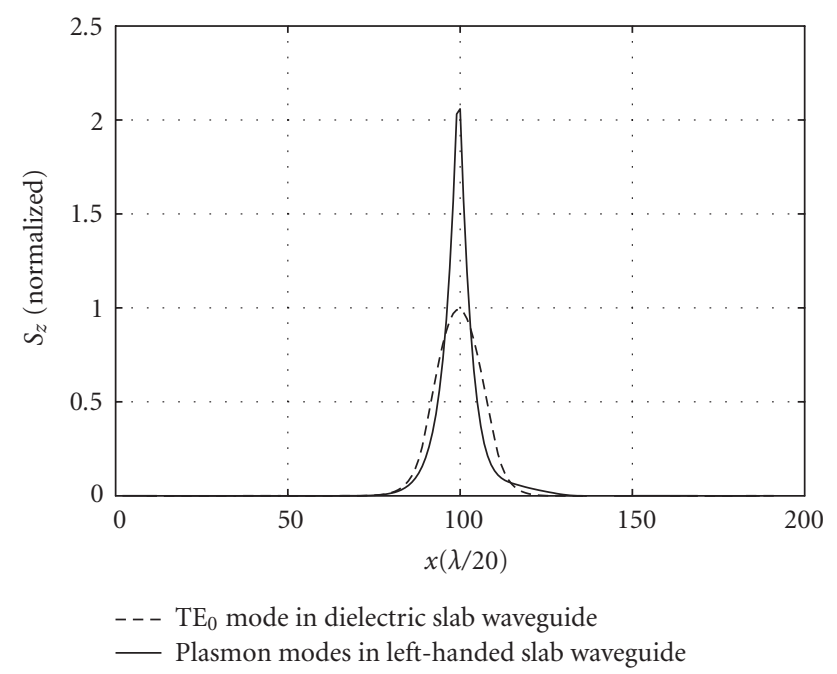

FIGURE 8: FDTD simulations show the power density distribution of the propagating guided waves in the dielectric slab waveguide and anisotropic $\mu$-negative slab waveguide (SW) of thickness $\lambda_{0}$, as depicted in Figure 6.

$\omega_{p}$ is defined as the plasma resonant frequency, and the medium exhibits negative permittivity and permeability for frequencies below $\omega<\omega_{p}$. The structure is truncated by using the split-field perfectly matched layer (PML), which can be conveniently combined with the Drude dispersion model of the permittivity and permeability in the LHM. The FDTD simulations shown in Figure 7 indicate that the proposed structure locally increases the power density of the guided wave at the core-cladding interface of the anisotropic $\mu$-negative SW. The steady-state field distributions of the guided $\mathrm{TE}_{0}$ mode of the dielectric slab waveguide and plasmon modes of the $\mu$-negative SW, depicted in Figure 8, indicate that the proposed signal detector can detect signals that are more than $3 \mathrm{~dB}$ weaker in comparison with their traditional counterparts. The anisotropic $\mu$-negative SW also supports undesirable guided modes with real transverse wavenumber. A simple overlap integral suggests that more than $95 \%$ of the incident power is coupled to the even and odd plasmon modes as indicated in Figures 9 and 10. 


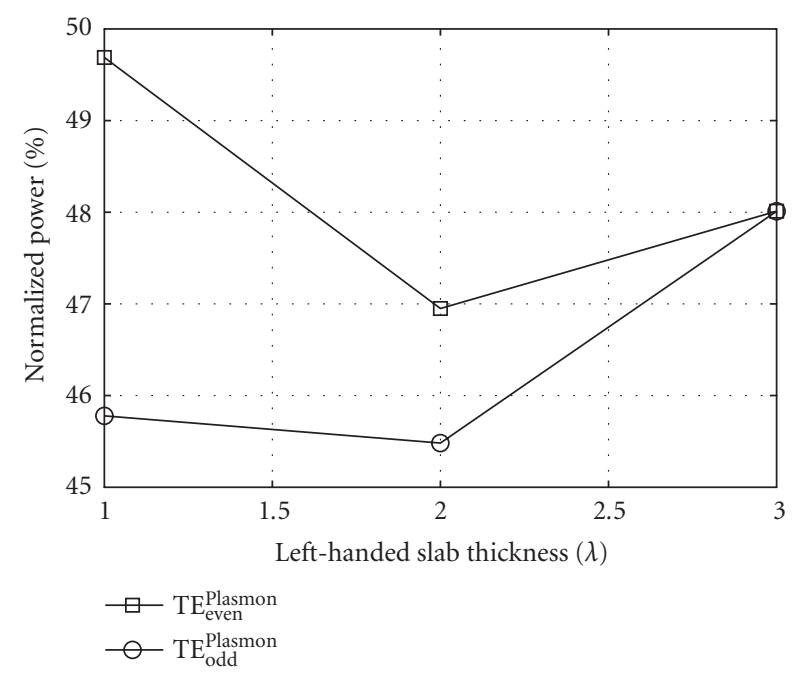

FIgURE 9: Percentage of the incident power coupled to the plasmon modes of the $\mu$-negative slab waveguide (SW).

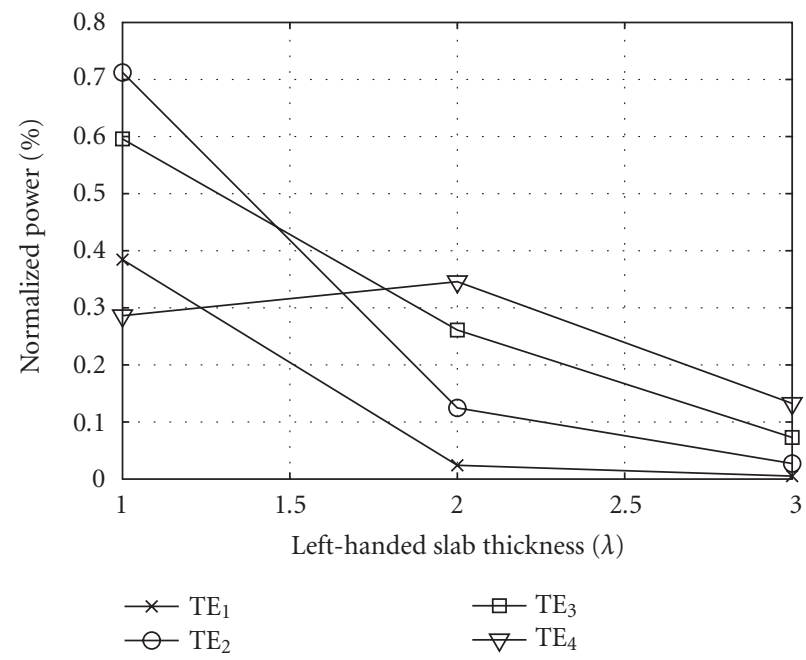

FIgURE 10: Percentage of the incident power coupled to the guided modes of the $\mu$-negative slab waveguide (SW) with real transverse wavenumbers.

In the proposed signal detector structure, the signal can be detected by a solid state probe, such as a photodiode, that is placed at the interface of the $\mu$-negative SW. The effective width of the probe changes the sensitivity of the signal detector. Increasing the effective width, $w$, of the photodiode decreases the effective width gain of the proposed structure, which is defined as the ratio of the total power detected in the proposed signal detector to that of a conventional detector. Figure 11 shows the effective width gain of the proposed structure as a function of the photodiode effective width.

Another potential device application of the anisotropic $\mu$-negative SW is in the design of a two-channel harmonic separator multiplexer. The structure of the proposed twochannel multiplexer is depicted in Figure 12. The operation mechanism of the proposed structure is as follows. At any operating frequency, the anisotropic $\mu$-negative slab waveg-

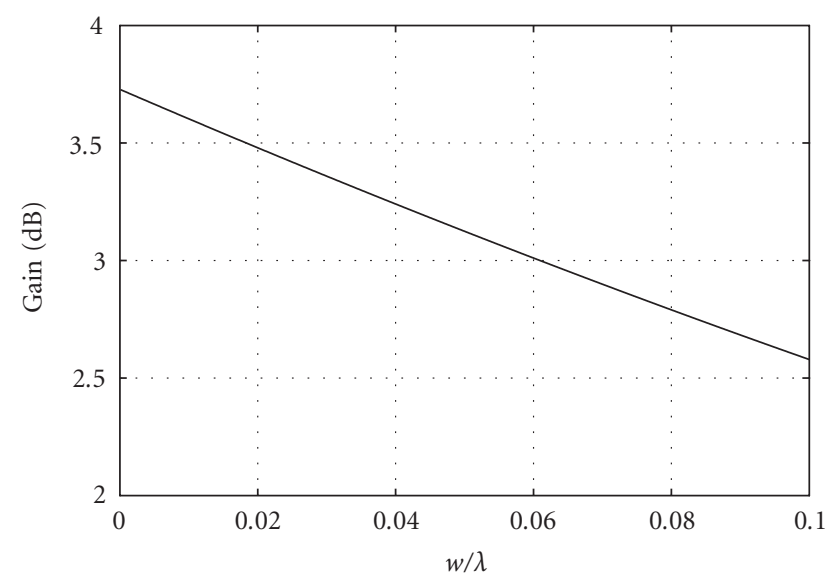

FIGURE 11: Effective width gain of the proposed signal detector as a function of the probe effective width, $w$, indicating that the sensitivity of the left-handed detector decreases as $w$ increases.

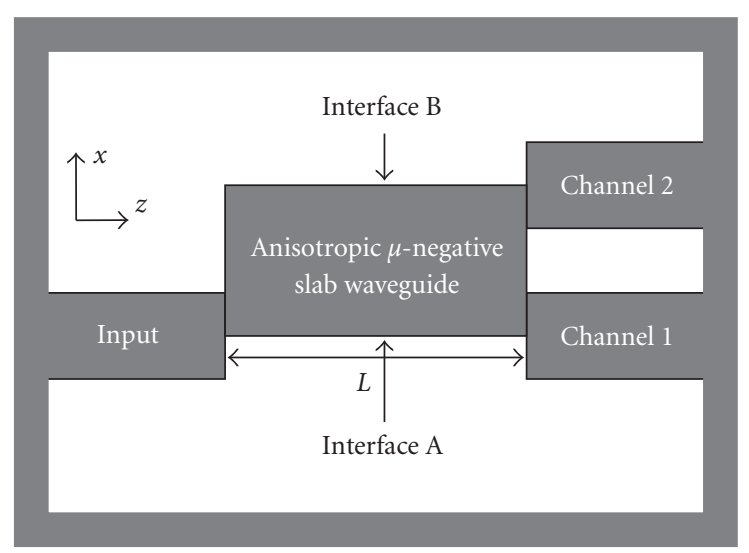

Figure 12: Schematic of the proposed two-channel multiplexer. The anisotropic $\mu$-negative slab waveguide has a thickness of $0.5 \lambda_{0}$, length of $L=6.25 \lambda_{0}$, with $\mu_{2}^{z z}=-1, \mu_{2}^{x x}=1$, and $\epsilon_{2}^{y y}=2.25$. The dielectric waveguides, located in the input, output channel one, and output channel two, have a thickness of $0.25 \lambda_{0}$ with $\epsilon=2.25$.

uide supports two guided modes with imaginary transverse wavenumbers, namely, even and odd plasmon modes. As the operating frequency increases, the propagation constants of the even and odd plasmon modes converge towards a common value. The difference between the propagation constants of the even and odd plasmon modes as a function of frequency is depicted in Figure 13. If the operating wavelength is greater than the anisotropic $\mu$-negative SW thickness, the even and odd plasmon modes interfere constructively at interface A of the structure in Figure 12, and destructively at interface B at the input waveguide junction. The structure can be designed to guide the fundamental frequency, $f_{0}$, to channel 2 and its harmonics to channel 1 . To do so, the propagating wave with fundamental wavelength $\lambda_{0}$ should interfere constructively at interface B of the structure in Figure 12, while interfere destructively at interface A at 


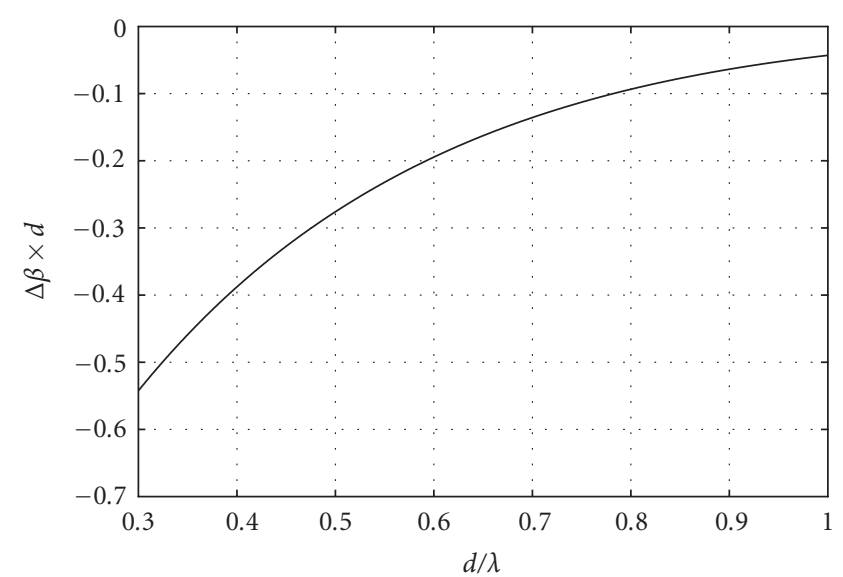

Figure 13: $\Delta$ propagation constants of even and odd plasmon modes of the $\mu$-negative slab waveguide (SW) as a function of the operating wavelength.

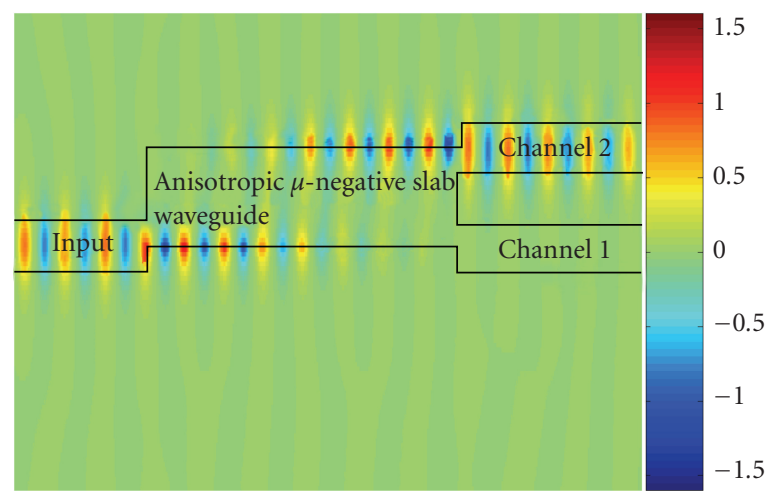

FIgURE 14: Proposed two-channel harmonic separator multiplexer directing the fundamental frequency to channel 2.

the output waveguide junction. The length of the anisotropic $\mu$-negative SW is found from $\left(\beta_{\text {even }}\left(f_{0}\right)-\beta_{\text {odd }}\left(f_{0}\right)\right) \times L=$ $\Delta \beta \times L=\pi$, which results in the constructive interference of the even and odd plasmon modes at interface B. Therefore, the fundamental frequency, $f_{0}$, is directed to channel 2 , whereas its harmonics, that interfere constructively at interface $\mathrm{A}$, are guided to channel 1 . As an example, consider a two-channel harmonic separator multiplexer that incorporates an anisotropic $\mu$-negative slab waveguide of width $t=0.5 \lambda_{0}$ and length $L=6.25 \lambda_{0}$. Figures 14 and 15 show the FDTD simulations of the proposed structure that directs the fundamental frequency, $f_{0}$, to channel 2 and its first harmonic, $2 f_{0}$, to channel 1 . Following the same mechanisms, other harmonics are also guided to channel 1 since at higher frequencies, $\Delta \beta$ becomes very small (see Figures 2 and 13).

\section{CONCLUSION}

The properties of different anisotropic left-handed slab waveguides are investigated. The existence of plasmon modes in various anisotropic structures is examined. It is shown that an anisotropic left-handed slab waveguide does not sup-

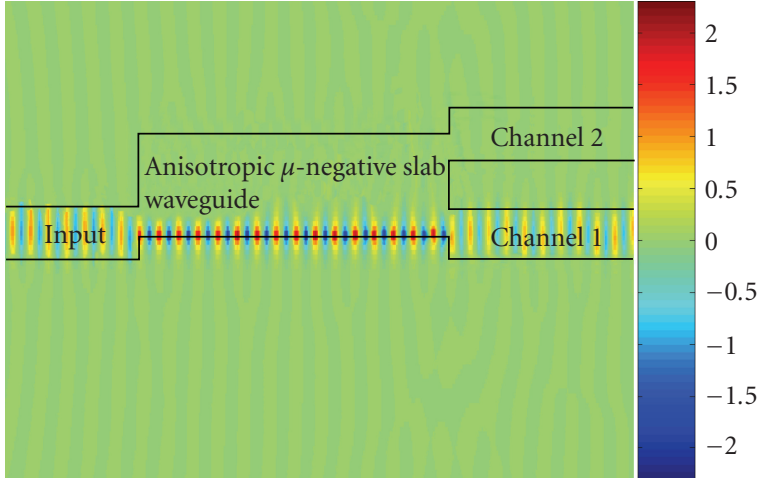

FIGURE 15: Proposed two-channel harmonic separator multiplexer directing the first harmonic of the fundamental frequency to channel 1.

port plasmon modes with imaginary transverse wavenumber. However, it is confirmed that anisotropic $\mu$-negative slab waveguides can support plasmon modes, which localizes the field at the interfaces of the waveguide. Two potential device applications of the anisotropic $\mu$-negative slab waveguide are identified. An FDTD program is developed to analyze the performance of the proposed devices.

\section{REFERENCES}

[1] V. Veselago, "The electrodynamics of substances with simultaneously negative values of $\varepsilon$ and $\mu$," Soviet Physics Uspekhi, vol. 10, no. 4, pp. 509-514, 1968.

[2] D. R. Smith, W. J. Padilla, D. C. Vier, S. C. Nemat-Nasser, and S. Schultz, "Composite medium with simultaneously negative permeability and permittivity," Physical Review Letters, vol. 84, no. 18, pp. 4184-4187, 2000.

[3] R. W. Ziolkowski, "Design, fabrication, and testing of double negative metamaterials," IEEE Transactions on Antennas and Propagation, vol. 51, no. 7, pp. 1516-1529, 2003.

[4] G. V. Eleftheriades, A. K. Iyer, and P. C. Kremer, "Planar negative refractive index media using periodically LC loaded transmission lines," IEEE Transactions on Microwave Theory and Techniques, vol. 50, no. 12, pp. 2701-2712, 2002.

[5] C. Caloz and T. Itoh, "Transmission line approach of lefthanded (LH) materials and microstrip implementation of an artificial LH transmission line," IEEE Transactions on Antennas and Propagation, vol. 52, no. 5, pp. 1159-1166, 2004.

[6] N. Engheta and R. W. Ziolkowski, "A positive future for double-negative metamaterials," IEEE Transactions on Microwave Theory and Techniques, vol. 53, no. 4, pp. 1535-1556, 2005.

[7] B. Wu, T. M. Grzegorczyk, Y. Zhang, and J. A. Kong, "Guided modes with imaginary transverse wave number in a slab waveguide with negative permittivity and permeability," Journal of Applied Physics, vol. 93, no. 11, pp. 9386-9388, 2003.

[8] I. V. Shadrivov, A. A. Sukhorukov, and Y. S. Kivshar, "Guided modes in negative-refractive-index waveguides," Physical Review E, vol. 67, no. 5, Article ID 057602, 4 pages, 2003.

[9] H. Salehi, S. K. Chaudhuri, and R. R. Mansour, "Unusual mode propagation characteristics of negative index slab waveguides," in Proceedings of IEEE International Symposium on Antennas and Propagation Society (ISAP '05), vol. 1A, pp. 721-724, Washington, DC, USA, July 2005. 
[10] A. Taflove and S. C. Hagness, Computational Electrodynamics: The Finite-Difference Time-Domain Method, Artech House, London, UK, 3rd edition, 2005.

[11] T. M. Grzegorczyk, Z. M. Thomas, and J. A. Kong, "Inversion of critical angle and brewster angle in anisotropic left-handed metamaterials," Applied Physics Letters, vol. 86, no. 25, Article ID 251909, 3 pages, 2005.

[12] T. M. Grzegorczyk, M. Nikku, X. Chen, B. I. Wu, and J. A. Kong, "Refraction laws for anisotropic media and their application to left-handed metamaterials," IEEE Transactions on Microwave Theory and Techniques, vol. 53, no. 4, pp. 14431450, 2005.

[13] D. R. Smith, P. Kolinko, and D. Schurig, "Negative refraction in indefinite media," Journal of the Optical Society of America $B$, vol. 21, no. 5, pp. 1032-1043, 2004.

[14] D. R. Smith, P. M. Rye, J. J. Mock, D. C. Vier, and A. F. Starr, "Enhanced diffraction from a grating on the surface of a negative-index metamaterial," Physical Review Letters, vol. 93, no. 13, Article ID 137405, 4 pages, 2004.

[15] V. A. Podolskiy and E. E. Narimanov, "Strongly anisotropic waveguide as a nonmagnetic left-handed system," Physical Review B, vol. 71, no. 20, Article ID 201101, 4 pages, 2005.

[16] L. Hu and S. T. Chui, "Characteristics of electromagnetic wave propagation in uniaxially anisotropic left-handed materials," Physical Review B, vol. 66, no. 8, Article ID 85108, 7 pages, 2002.

[17] D. R. Smith and D. Schurig, "Electromagnetic wave propagation in media with indefinite permittivity and permeability tensors," Physical Review Letters, vol. 90, no. 7, Article ID 077405, 4 pages, 2003. 

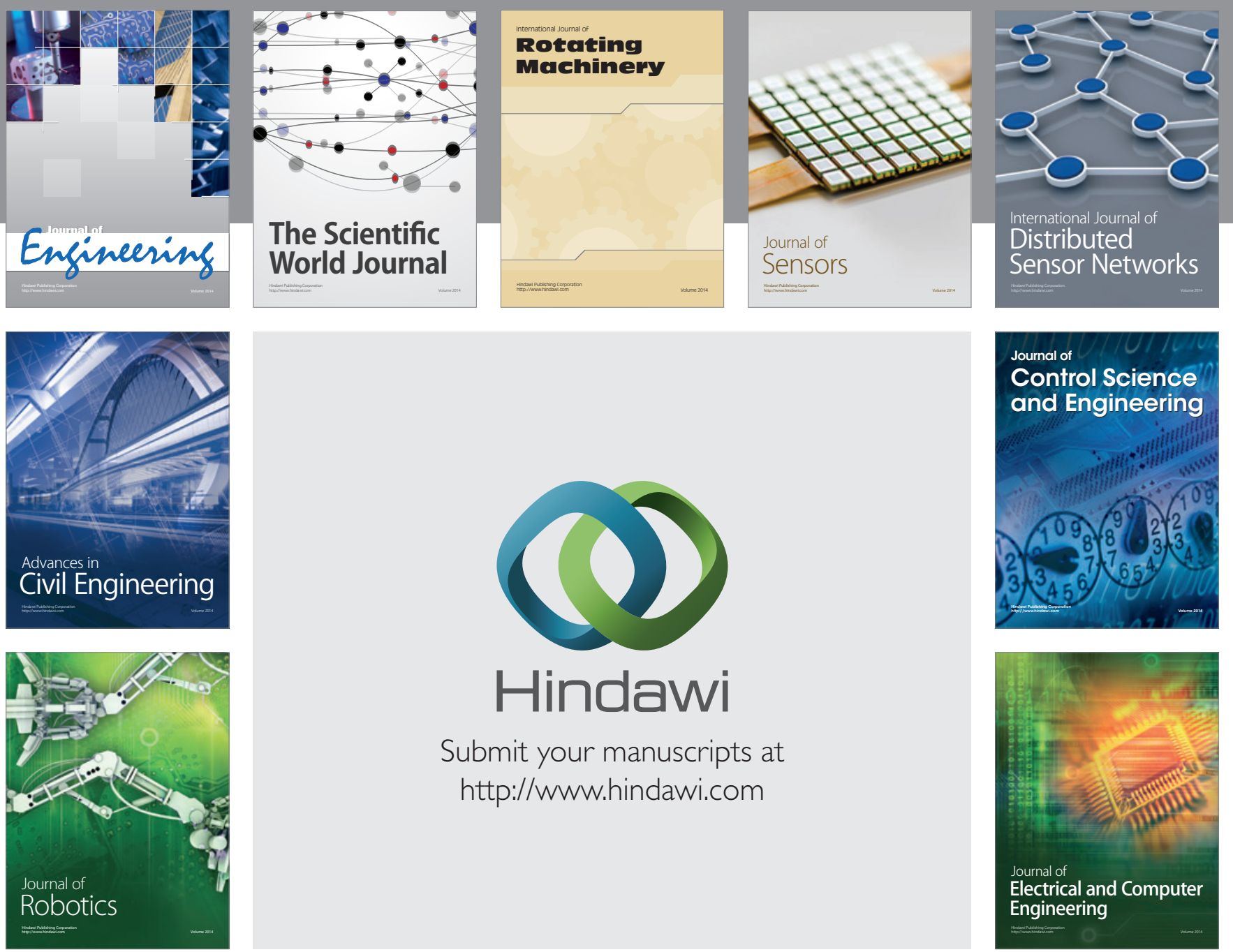

Submit your manuscripts at

http://www.hindawi.com
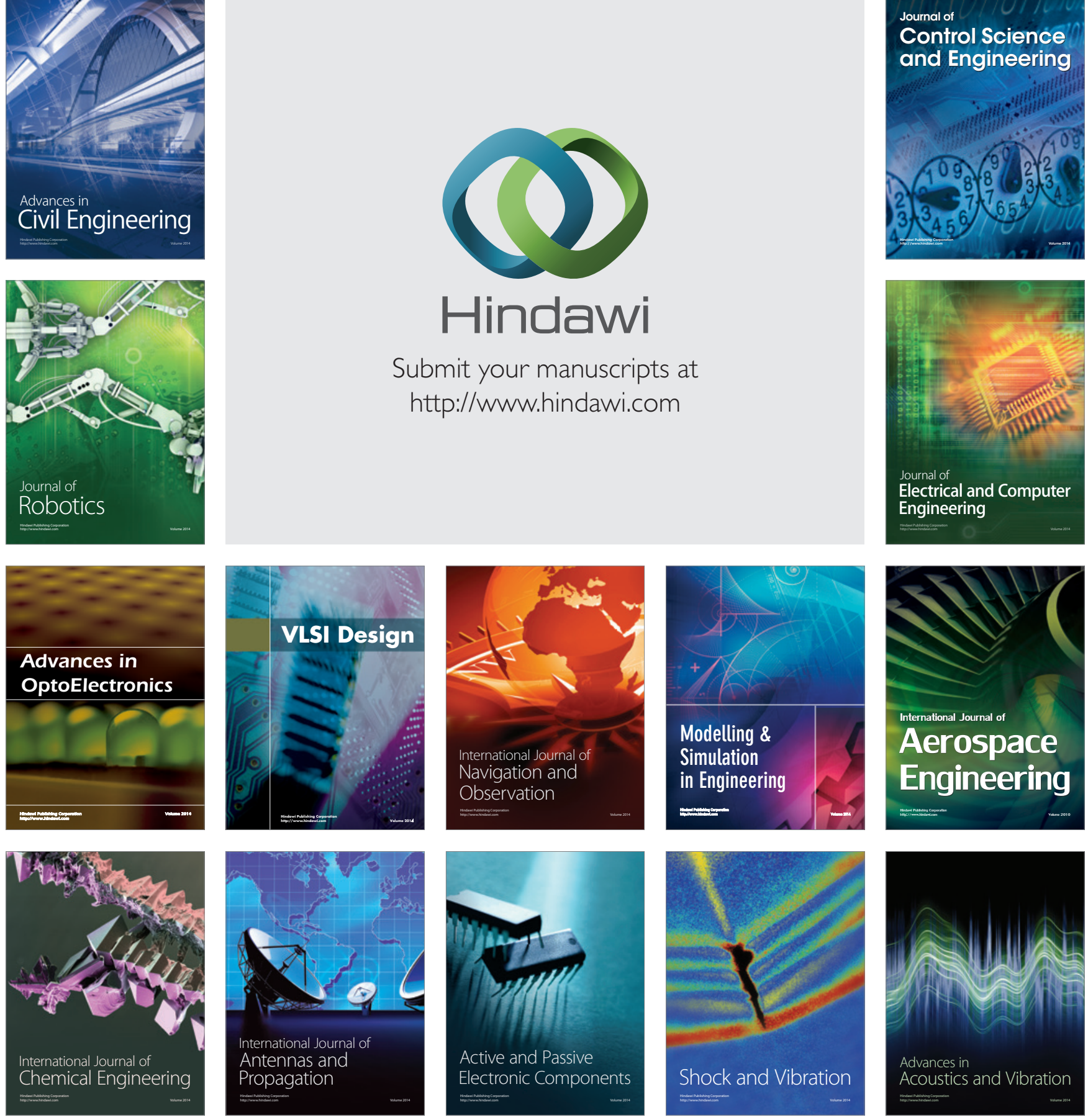